\title{
ON PLANAR POLYNOMIAL VECTOR FIELDS WITH ELEMENTARY FIRST INTEGRALS
}

\author{
JAUME LLIBRE ${ }^{1}$, CHARA PANTAZI $^{2}$ AND SEBASTIAN WALCHER ${ }^{3}$
}

\begin{abstract}
We show that under rather general conditions a polynomial differential system having an elementary first integral already must admit a Darboux first integral, and we explicitly characterize the vector fields in this class. We also investigate some exceptional cases, i.e. equations admitting an elementary first integral but not a Darboux first integral. In particular we provide a rather detailed discussion of exceptional elementary first integrals built from algebraic functions of degree two or three.
\end{abstract}

\section{INTRODUCTION AND SURVEY OF RESULTS}

In the present note we discuss a polynomial vector field

$$
X=P \frac{\partial}{\partial x}+Q \frac{\partial}{\partial y}, \quad \text { briefly } X=\left(\begin{array}{l}
P \\
Q
\end{array}\right)
$$

in $\mathbb{C}^{2}$, and the associated ordinary differential equation

$$
\begin{aligned}
& \dot{x}=P(x, y), \\
& \dot{y}=Q(x, y) .
\end{aligned}
$$

Our focus is on systems which admit a first integral that is elementary over the differential field $\mathbb{K}=\mathbb{C}(x, y)$. (The pertinent notions will be introduced below; in particular we collect some basic definitions and facts in an appendix.) Our approach is based on the classical work [10] by Prelle and Singer. We recall some of their main results, specialized to the rational function field.

Theorem 1. (See Prelle and Singer [10])

(a) If the polynomial vector field $X$ admits an elementary first integral then there exist an integer $m \geq 0$, algebraic functions $v, u_{1}, \ldots, u_{m}$ over $\mathbb{K}$ and nonzero constants $c_{1}, \ldots, c_{m} \in \mathbb{C}$ such that

$$
X(v)+\sum_{i=1}^{m} c_{i} \frac{X\left(u_{i}\right)}{u_{i}}=X\left(v+\sum_{i=1}^{m} c_{i} \log \left(u_{i}\right)\right)=0,
$$

but $v+\sum_{i=1}^{m} c_{i} \log \left(u_{i}\right)$ is not constant. The $c_{i}$ may be chosen linearly independent over the rational numbers $\mathbb{Q}$. 
(b) If the vector field $X$ admits an elementary first integral then it admits an integrating factor of the special form

$$
\mu=f_{1}^{-d_{1}} \cdots f_{r}^{-d_{r}}
$$

with irreducible and pairwise relatively prime polynomials $f_{1}, \ldots, f_{r}$, and exponents $d_{1}, \ldots, d_{r} \in \mathbb{Q}$.

The second statement includes the case when a rational first integral exists; in the particular case of a polynomial first integral there may be a constant integrating factor. Note that the existence of a first integral $v+\sum_{i=1}^{m} c_{i} \log \left(u_{i}\right)$ for (1) is equivalent to the existence of a first integral

$$
\exp (v) \cdot \prod u_{i}^{c_{i}} .
$$

The aim of the present paper is to gain more insight into the nature of the algebraic functions $v$ and $u_{i}$, and to obtain a structural characterization of the polynomial vector fields which admit such a first integral. Thus we prove that, whenever the trace of $v$ is not constant, or the norm of some $u_{i}$ is not constant then the existence of a first integral (4) already implies the existence of a Darboux first integral (i.e., a first integral of this type with rational functions $v$ and $u_{i}$ ); this yields a precise characterization of the vector fields which admit this first integral. With regard to the exceptional cases we prove some general results about the simplest one (the case of a first integral (2) with $m=1$ ), and discuss some classes of examples. Our results indicate that the name "exceptional" is justified.

Before we state the results in detail, we briefly recall the general setting, with a particular focus on integrating factors of type (3). By Singer [13] and Christopher [3], the vector field (1) admits a Liouvillian first integral if it admits an integrating factor of type (3) with complex exponents $d_{i}$. Moreover, there exists a Liouvillian first integral if and only if there exists a Darboux integrating factor. The existence of a Liouvillian first integral is not always associated to the existence of some invariant algebraic curve, see [7]. The special structure of polynomial vector fields admitting an integrating factor of type (3) (with exponents $d_{i} \in \mathbb{C}$ ) has been discussed in a number of recent papers, e.g. $[2,9,4,5,6]$. We will use some of these results below.

Returning to polynomial vector fields with elementary first integrals, the particular vector fields which even admit a rational first integral form a special class. On the one hand such vector fields are easy to construct by forming the Hamiltonian of a rational function and multiplying it by a suitable factor to obtain a polynomial (with relatively prime entries, if desired). On the other hand it may be problematic to decide for a given polynomial vector field whether it admits a rational first integral. There are several algorithms to compute rational first integrals of a given polynomial vector field when degree bounds are given; see e.g. Bostan et al. [1]. But determining such degree bounds a priori is an unresolved problem. 
We will frequently require that a vector field under consideration does not admit a rational first integral. This implies in particular (as will be noted in Section 3 below) that we may assume that $v$ is not constant, $m>0$ and the $u_{i}$ are not constant in (2).

Our principal result states that under rather weak assumptions the existence of an elementary first integral will already imply the existence of a Darboux first integral.

Theorem 2. Let the vector field $X$ given in (1) admit the elementary first integral $v+\sum_{i=1}^{m} c_{i} \log \left(u_{i}\right)$, where $m>0$ and $v, u_{1}, \ldots, u_{m}$ are nonconstant algebraic functions over $\mathbb{K}=\mathbb{C}(x, y)$, and furthermore $c_{1}, \ldots, c_{m}$ are complex constants linearly independent over $\mathbb{Q}$. If $X$ does not admit a rational first integral then the following hold.

(a) If some $u_{j}$ has non-constant norm, or if $v$ has non-constant trace, then $X$ admits a Darboux first integral.

(b) Moreover, if (3) is an integrating factor for $X$, with some $u_{j}$ having nonconstant norm, then it is uniquely determined (up to a nonzero complex factor) and all $d_{i}$ are nonnegative integers.

We will prove this in Section 3. Moreover, given the setting of statement (a) of Theorem 2, the corresponding polynomial vector fields can be determined explicitly, up to multiplication by a polynomial function, by invoking a result of Chavarriga et al. [2]; see Theorem 4 and Corollary 1.

Theorem 2 leaves the problem to investigate "exceptional" cases admitting an elementary first integral (2) with all $u_{i}$ of constant norm, $v$ of constant trace. In the present work we restrict our attention to the (presumably) simplest case $m=1$. One may then take $c_{1}=1$ with no further loss of generality. Thus we assume a relation

$$
X(v)+\frac{X(u)}{u}=0,
$$

with $u$ and $v$ non-constant algebraic functions over $\mathbb{K}$ (but not both in $\mathbb{K}$ ), with $u$ of constant norm and $v$ of constant trace. In Section 4 we will prove:

Proposition 1. Let relation (5) be given, and assume that $X$ admits no rational first integral.

(a) The intersection $\mathbb{K}[u] \cap \mathbb{K}[v]$ is nontrivial, i.e. $\neq \mathbb{K}$.

(b) If the degree of $\mathbb{K}[v]$ over $\mathbb{K}$ is a prime number, then $\mathbb{K}[v] \subseteq \mathbb{K}[u]$. If both degrees are prime numbers then $\mathbb{K}[v]=\mathbb{K}[u]$.

We proceed to discuss the cases when both $u$ and $v$ are contained in a degree two extension, respectively a degree three extension of $\mathbb{K}$. For the quadratic setting we give a complete characterization as follows.

Theorem 3. Assume relation (5) with $u$ of constant norm and $v$ of constant trace, both contained in a degree two extension of $\mathbb{K}$, but neither contained in $\mathbb{K}$. Then, with no loss of generality, one may take $u$ and $v$ to satisfy

$$
u^{2}+2 g \cdot u+1=0 \text { and } v=b(g+u)
$$


with a non-constant rational function $g$ and a nonzero rational function $b$.

(a) There is a rational function s such that

$$
\begin{aligned}
& P=-s \cdot\left(\left(g^{2}-1\right) b_{y}+(b g-1) g_{y}\right), \\
& Q=s \cdot\left(\left(g^{2}-1\right) b_{x}+(b g-1) g_{x}\right),
\end{aligned}
$$

defines a polynomial vector field. If one requires $P$ and $Q$ to have relatively prime entries then $s$ is unique up to a factor in $\mathbb{C}^{*}$.

(b) This vector field admits the elementary first integral $v+\log u$ and the integrating factor $\left(\sqrt{g^{2}-1} \cdot s\right)^{-1}$.

(c) If this vector field admits a Darboux first integral then it admits a rational first integral.

For the proof see Section 4; we also give some examples which do not admit a rational first integral.

Turning to degree three extensions, matters become more complicated. Thus let $\mathbb{F}=\mathbb{K}[u]=\mathbb{K}[v]$ be a degree three extension, with $v$ of trace zero and $u$ of norm one (with no loss of generality), and consider a polynomial vector field $X$ admitting the elementary first integral (5). The following results are also proven in Section 4.

Proposition 2. Let $\mu$ be the integrating factor of $X$ (which is unique up to a nonzero scalar). Then $\mathbb{F}=\mathbb{K}[\mu]$ and there exists $g \in \mathbb{K}$ such that

$$
\mu^{3}-g=0
$$

Note that $g$ is not a cube in $\mathbb{K}$, and that the field extension $\mathbb{F}: \mathbb{K}$ is a cyclic Galois extension of degree three. A general element of $\mathbb{F}$ has the form

$$
w:=a+b \cdot \mu+c \cdot \mu^{2} ; \quad a, b, c \in \mathbb{K},
$$

and by Hilbert's Theorem 90 we may write

$$
u=\frac{\sigma(w)}{w},
$$

for some nonzero $w$, while the trace zero element has the form

$$
v=r \cdot \mu+s \cdot \mu^{2} ; \quad r, s \in \mathbb{K} .
$$

Proposition 3. Let $w, u$ and $v$ be as above, and denote by $N(w)$ the norm of $w$. Then the following hold.

(a) For every derivation $Y$ of $\mathbb{K}$ one has

$$
\frac{Y(u)}{\mu \cdot u}=\frac{1}{N(w)} A_{Y}+\frac{\mu}{N(w)} B_{Y}
$$


with rational functions $A_{Y}$ and $B_{Y}$. Explicitly

$$
\begin{aligned}
B_{Y}= & g b c^{2}\left(\frac{Y(c)}{c}-\frac{Y(b)}{b}+\frac{1}{3} \frac{Y(g)}{g}\right) \\
& +a b^{2}\left(\frac{Y(b)}{b}-\frac{Y(a)}{a}+\frac{1}{3} \frac{Y(g)}{g}\right) \\
& +a^{2} c\left(\frac{Y(a)}{a}-\frac{Y(c)}{c}-\frac{2}{3} \frac{Y(g)}{g}\right) .
\end{aligned}
$$

(b) There exists a nontrivial polynomial vector field $X$ which admits the first integral $H:=v+\log u$ if and only if the identity

$$
Y(s)+\frac{2}{3} s \cdot \frac{Y(g)}{g}+\frac{1}{N(w)} B_{Y}=0
$$

holds for all derivations $Y$ of $\mathbb{K}$.

(c) Given any $u \in \mathbb{F}$ with norm one, there exists at most one rational function $s$ such that $H$ (with $v=r \cdot \mu+s \cdot \mu^{2}$ and arbitrary $r \in \mathbb{K}$ ) is a first integral for some nontrivial polynomial vector field.

This result provides strong restrictions to possible exceptional elementary first integrals of polynomial vector fields. Even more, it provides obstructions to their existence. We discuss examples in Section 4; it should be noted that we could not find a polynomial vector field which admits an exceptional first integral of this type but not a rational one.

\section{NOTIONS AND KNOWN FACTS}

We start by recalling some notions and facts (see the appendix for more). We sketch some proofs, for easy reference and the reader's convenience.

Definition 1. We call a function

$$
\exp (R) \cdot \prod S_{i}^{c_{i}}
$$

with rational functions $R$ and $S_{i}$ and complex constants $c_{i}$, a Darboux function.

The notions of Darboux first integral and Darboux integrating factor are then self-explaining.

Lemma 1. Let (1) be given, and $H$ an analytic first integral of this vector field on an open set $U$. If the nonzero polynomial vector field

$$
\widetilde{X}=\widetilde{P} \frac{\partial}{\partial x}+\widetilde{Q} \frac{\partial}{\partial y}
$$

also admits the first integral $H$ on $U$ then there exists a rational function $R$ such that $\widetilde{X}=R \cdot X$.

Proof. There exist analytic $\mu$ and $\widetilde{\mu}$ such that

$$
\mu X=X_{H}=-H_{y} \partial / \partial x+H_{x} \partial / \partial y=\tilde{\mu} \widetilde{X},
$$


see Section 5 . Hence one has $\widetilde{X}=R \cdot X$ on a nonempty open subset of $\mathbb{C}^{2}$, and (e.g.) $R=\widetilde{P} / P$ shows that $R$ is rational.

The vector fields with non-constant Darboux integrating factor (3) (with fixed polynomials and exponents) form a linear space $\mathcal{F}$. The so-called trivial vector fields admitting (3) form a subspace $\mathcal{F}^{0} \subseteq \mathcal{F}$. To construct these, start with an arbitrary polynomial $g$, define

$$
Z_{g}=\text { Hamiltonian vector field of } g /\left(f_{1}^{d_{1}-1} \cdots f_{r}^{d_{r}-1}\right) .
$$

Then, according to [5], the trivial vector field

$$
f_{1}^{d_{1}} \cdots f_{r}^{d_{r}} \cdot Z_{g}=f \cdot X_{g}-\sum_{i=1}^{r}\left(d_{i}-1\right) g \frac{f}{f_{i}} \cdot X_{f_{i}}
$$

(with $X_{h}$ denoting the Hamiltonian vector field of a function $h$ ) is polynomial and admits (3) by construction.

Proposition 4. If $d_{1}, \ldots, d_{r}$ are rational numbers then every element of $\mathcal{F}^{0}$ admits a rational (hence elementary) first integral.

Proof. Some integer power of $g /\left(f_{1}^{d_{1}-1} \cdots f_{r}^{d_{r}-1}\right)$ is a rational function.

We remark that one has $\mathcal{F}=\mathcal{F}^{0}$ whenever the geometry of the underlying curves (including the line at infinity) is nondegenerate; see $[4,5]$. In such a case the above Proposition applies. Next we recall a precise description of polynomial vector fields which admit a Darboux first integral.

Theorem 4. (See Chavarriga et al. [2], and [9].) Let

$$
H(x, y)=f_{1}^{\lambda_{1}} \cdots f_{r}^{\lambda_{r}} \exp \left(g /\left(f_{1}^{n_{1}} \cdots f_{r}^{n_{r}}\right)\right)
$$

be a Darboux function with $\lambda_{1}, \cdots, \lambda_{r} \in \mathbb{C}, n_{1}, \cdots, n_{r} \in \mathbb{N} \cup\{0\}$ and $g \in$ $\mathbb{C}[x, y]$ coprime with $f_{i}$ whenever $n_{i} \neq 0$. Then $H$ is a first integral of the polynomial vector field

$$
\widehat{X}=\prod_{k=1}^{r} f_{k}^{n_{k}+1} \cdot\left(\sum_{k=1}^{r} \lambda_{k} X_{f_{k}} / f_{k}+Z_{g}^{\left(n_{1}+1, \ldots, n_{r}+1\right)}\right)
$$

which, in turn, admits the integrating factor $\prod_{k=1}^{r} f_{k}^{-\left(n_{k}+1\right)}$. Moreover, any polynomial vector field admitting the first integral $H$ admits a rational integrating factor.

Sketch of proof. One verifies (as in [9]) directly that

$$
\widehat{X}=\left(\prod_{l=1}^{r} f_{l}^{n_{l}}\right) \sum_{i=1}^{r} \lambda_{i}\left(\prod_{\substack{j=1 \\ j \neq i}}^{r} f_{j}\right) X_{f_{i}}-g \sum_{i=1}^{r} n_{i}\left(\prod_{\substack{j=1 \\ j \neq i}}^{r} f_{j}\right) X_{f_{i}}+\left(\prod_{j=1}^{r} f_{j}\right) X_{g}
$$


admits the first integral $H$. Comparison with equation (8) shows that $\widehat{X}$ admits a representation as stated. The remaining assertions are obvious, resp. follow from Lemma 1.

Corollary 1. If the vector field (1) admits a Darboux first integral but not a rational first integral then it admits (up to constant multiples) a unique integrating factor, and this integrating factor is rational.

As for a converse, we have the following result which easily follows from a theorem due to Rosenlicht [12].

Proposition 5. If the vector field (1) admits a rational integrating factor then it admits a Darboux first integral.

Proof. There exists a rational function $\mu$ such that $\mu \cdot X$ is the Hamiltonian vector field of some analytic function $H$, hence $H_{x}$ and $H_{y}$ are rational. By Rosenlicht [12], Theorem 3, there exist $u_{i}$ and $v$ in $\mathbb{K}$, and $c_{i} \in \mathbb{C}$ such that $H=v+\sum c_{i} \log u_{i}$; thus exp $H$ is a Darboux first integral.

\section{Elementary and Darboux first integrals}

In this section we will prove Theorem 2. By Theorem 1 we may assume there exists an elementary first integral for $X$ of the form (2), which is built from algebraic functions over $\mathbb{K}=\mathbb{C}(x, y)$, and their logarithms.

Given an algebraic function $w$, we denote its minimal polynomial by

$$
M_{w}(T):=T^{d}+\sum_{i=1}^{d} g_{i} T^{d-i} \in \mathbb{K}[T] .
$$

Here $-g_{1}$ is the trace of $w$ and $(-1)^{d} g_{d}$ is the norm of $w$. The other zeros of $M_{w}$ (in a suitable extension field $\mathbb{F}$ of $\mathbb{K}$ ) are called the conjugates of $w$. (For algebraic notions and facts the reader may consult e.g. Lang [8].) The following observation is well-known.

Lemma 2. If an algebraic function $w$ is a first integral of (1) then all nonconstant coefficients of its minimal polynomial are first integrals of (1).

Proof. Indeed, $X(w)=0$ implies

$$
\begin{aligned}
0 & =X\left(M_{w}(w)\right) \\
& =X(w) \cdot\left(d w^{d-1}+\sum_{i=1}^{d-1}(d-i) g_{i} w^{d-i-1}\right)+\sum_{i=1}^{d} X\left(g_{i}\right) w^{d-i} \\
& =\sum_{i=1}^{d} X\left(g_{i}\right) w^{d-i}
\end{aligned}
$$

which forces all $X\left(g_{i}\right)=0$ by uniqueness of the minimal polynomial.

We note one more property that may help in computations.

Proposition 6. If the polynomial vector field $X$ admits an elementary first integral (2) then it admits an algebraic integrating factor $\mu$ in $\mathbb{F}:=$ $\mathbb{K}\left[v, u_{1}, \ldots, u_{m}\right]$. Moreover, if $\widehat{\mathbb{F}}$ is a normal extension of $\mathbb{F}$ with degree $[\widehat{\mathbb{F}}: \mathbb{K}]=n$ then $\mu^{n} \in \mathbb{K}$. 
Proof. (i) We first show that the partial derivatives of $v$ and all $u_{i}$ are contained in $\mathbb{K}\left[v, u_{1}, \ldots, u_{m}\right]$. Indeed, let

$$
v^{e}+\sum_{i=1}^{e} h_{i} v^{e-i}=0 ; \quad h_{1}, \ldots, h_{e} \in \mathbb{K} .
$$

Differentiate to obtain

$$
0=v_{x} \cdot\left(e v^{e-1}+\sum_{i=1}^{e-1} h_{i}(e-i) v^{e-i-1}\right)+\sum_{i=1}^{e} h_{i, x} v^{e-i}
$$

and hence $v_{x} \in \mathbb{K}(v)=\mathbb{K}[v]$. The same proof applies to $v_{y}$, and by the same arguments we obtain that $u_{i, x}$ and $u_{i, y}$ lie in $\mathbb{K}\left[u_{i}\right]$ for $1 \leq i \leq m$.

(ii) Thus, setting

$$
H:=v+\sum \log \left(u_{i}\right)
$$

we see that $H_{x}$ and $H_{y}$ are contained in $\mathbb{F}$. Since $H$ is a first integral for $X$, we have

$$
H_{y}=\mu \cdot P, \quad-H_{x}=\mu \cdot Q
$$

with some analytic function $\mu$. Since $H_{x}, H_{y}, P, Q$ are all contained in $\mathbb{F}$, the same must hold for $\mu$.

(iii) The last statement follows from the proof of Proposition 2 in Prelle and Singer [10]; note that the degree of $\widehat{\mathbb{F}}$ over $\mathbb{K}$ is the order of the Galois group.

Since we are interested in vector fields which do not admit a rational first integral, we may assume that the vector field $X$ given in (1) admits an elementary first integral (2) with $m>0$; equivalently we may require

$$
X\left(\exp (v) \cdot \prod_{i=1}^{m} u_{i}^{c_{i}}\right)=0
$$

with $m>0$. We may and will assume that the $c_{i}$ are linearly independent over the rational number field $\mathbb{Q}$.

Proof of Theorem 2. (i) Let $\mathbb{F}$ be the smallest normal extension of $\mathbb{K}$ which contains $v$ and all $u_{i}$, and denote its Galois group by $G$. Since $\mathbb{K}$ automorphisms of $\mathbb{F}$ commute with derivations (see e.g. Rosenlicht [11], Proposition and p. 156), we also have

$$
X(\sigma(v))+\sum_{i=1}^{m} c_{i} \frac{X\left(\sigma\left(u_{i}\right)\right)}{\sigma\left(u_{i}\right)}=0,
$$

for all $\sigma \in G$, and summation yields

$$
X\left(\sum_{\sigma \in G} \sigma(v)\right)+\sum_{i=1}^{m} c_{i} \frac{X\left(\prod_{\sigma \in G} \sigma\left(u_{i}\right)\right)}{\prod_{\sigma \in G} \sigma\left(u_{i}\right)}=0 .
$$


Since $R:=\sum_{\sigma \in G} \sigma(v)$ is a positive integer multiple of the trace of $v$, and $S_{i}:=\prod_{\sigma \in G} \sigma\left(u_{i}\right)$ is a power of the norm of $u_{i}$ with a positive integer exponent, $1 \leq i \leq m$, this identity involves only rational functions. ${ }^{1}$ In particular, whenever $R+\sum c_{i} \log S_{i} \neq$ const., equivalently $\exp (R) \cdot \prod S_{i}^{c_{i}} \neq$ const., we have a Darboux first integral. Note that whenever all $u_{i}$ have constant norm then the trace of $v$ must also be constant since there exists no rational first integral by assumption.

(ii) We show that $\exp (R) \cdot \prod S_{i}^{c_{i}} \neq$ const. whenever some $u_{k}$ has nonconstant norm. Thus let $p$ be an irreducible polynomial and $m_{k}$ a nonzero integer such that $S_{k}=p^{m_{k}} \cdot \widetilde{S}_{k}$, with $p$ dividing neither numerator nor denominator of $\widetilde{S}_{k}$. Likewise we have $S_{i}=p^{m_{i}} \cdot \widetilde{S}_{i}$, with an integer $m_{i}$ and $p$ dividing neither numerator nor denominator of $\widetilde{S}_{i}$, for all $i \neq k$.

As a preliminary step we show that $\prod S_{i}^{c_{i}} \neq$ const. Due to the linear independence of the $c_{i}$ over $\mathbb{Q}$ and $m_{k} \neq 0$ we have

$$
\prod S_{i}^{c_{i}}=p^{\sum c_{i} m_{i}} \cdot \prod \widetilde{S}_{i}^{c_{i}}, \quad \sum c_{i} m_{i} \neq 0
$$

and it suffices to show that

$$
p \cdot S^{*}:=p \cdot \prod \widetilde{S}_{i}^{e_{i}} \neq \text { const., } \quad \text { with } e_{i}:=c_{i} / \sum c_{j} m_{j} .
$$

There exists $\left(x_{0}, y_{0}\right) \in \mathbb{C}^{2}$ be such that $p\left(x_{0}, y_{0}\right)=0$ and $S^{*}$ is analytic and does not vanish in this point. Thus $p \cdot S^{*}$ has a zero in $\left(x_{0}, y_{0}\right)$ but is not identically zero.

We next show the full assertion. Let $R=p^{\ell} \cdot \widetilde{R}$, with $p$ not dividing numerator or denominator of $\widetilde{R}$. Whenever $\ell \geq 0$ the argument from above still works to prove non-constancy. Thus assume $\ell<0$ and consider

$$
\begin{aligned}
R^{*} & :=p^{-\ell}\left(R+\sum c_{i} \log S_{i}\right) \\
& =\widetilde{R}+\left(\sum c_{i} m_{i}\right) p^{-\ell} \log p+p^{-\ell} \sum c_{i} \log \widetilde{S}_{i} .
\end{aligned}
$$

There exists $\left(x_{0}, y_{0}\right) \in \mathbb{C}^{2}$ at which $\widetilde{R}$ and all $\widetilde{S}_{i}$ are analytic and nonzero but $p\left(x_{0}, y_{0}\right)=0$. Let $\gamma(t)=\left(x_{0}, y_{0}\right)+t \cdot a$, with $a \in \mathbb{C}^{2}$ parameterize a transversal line segment to the curve defined by $p=0$. Then standard growth estimates yield

$$
\lim _{t \rightarrow 0} R^{*}(t)=\widetilde{R}(0) \neq 0 \text {, thus }\left|R+\sum c_{i} \log S_{i}\right| \rightarrow \infty \text { as } t \rightarrow 0,
$$

and non-constancy follows as asserted. Thus part (a) is proven.

(iii) The assertion of part (b) follows by Theorem 4 and Corollary 1 .

\footnotetext{
${ }^{1}$ The positive integer in question is the degree $[\mathbb{F}: \widehat{\mathbb{F}}]$, with $\widehat{\mathbb{F}} \subset \mathbb{F}$ denoting the splitting field of $v$, respectively of $u_{i}$.
} 


\section{The Simplest EXCEPTIONAL SETting}

In this section we will discuss exceptional vector fields which, by definition, admit an elementary first integral but not a Darboux first integral. We restrict attention to the simplest setting, starting from a first integral $v+$ $\log u$ of $X$ as in relation (5), with $u$ and $v$ nonconstant and algebraic over $\mathbb{K}$ (but not both in $\mathbb{K}$ ), with $u$ of constant norm and $v$ of constant trace. Since $u$ may be changed to $\alpha \cdot u$ with some $\alpha \in \mathbb{C}^{*}$ and $v$ may be changed to $v+\beta$ with some $\beta \in \mathbb{C}$, we may assume that $u$ has norm one and $v$ has trace zero.

4.1. Some generalities. In this subsection we prove that $\mathbb{K}[u] \cap \mathbb{K}[v] \neq \mathbb{K}$ under the assumptions of Proposition 1. We first remark that neither $u$ nor $v$ are elements of $\mathbb{K}$, since this would imply $u \in \mathbb{C}$ (resp. $v \in \mathbb{C}$ ) and the existence of a rational first integral follows from Lemma 2.

The assertion of Proposition 1(a) is then a direct consequence of the following general fact; note that $X(u) / u$ and $X(v)$ are contained in the intersection due to (5).

Lemma 3. Let $s \neq 0$ be algebraic over $\mathbb{K}$ with constant norm, $Y$ a derivation of $\mathbb{K}[s]$ extending a derivation of $\mathbb{K}$, and $Y(s) / s \in \mathbb{K}$. Then $Y(s)=0$, hence $s$ is constant unless $Y$ admits a rational first integral.

Proof. The minimal polynomial of $s$ yields a relation

$$
s^{m}+g_{1} s^{m-1}+\cdots+g_{m-1} s+g_{m}=0
$$

with all $g_{i} \in \mathbb{K}\left(g_{0}:=1\right)$ and constant $g_{m}$. Applying the derivation yields

$$
0=Y(s) \cdot \sum_{i=1}^{m} g_{i-1}(m-i+1) s^{m-i}+\sum_{i=1}^{m-1} Y\left(g_{i}\right) s^{m-i},
$$

in view of $Y\left(g_{m}\right)=0$. Letting $Y(s)=h \cdot s$ with $h \in \mathbb{K}$, one finds

$$
m h s^{m}+\sum_{i=1}^{m-1}\left((m-i) h g_{i}+Y\left(g_{i}\right)\right) s^{m-i}=0 .
$$

On the other hand

$$
m h s^{m}+\sum_{i=1}^{m} m h g_{i} s^{m-i}=0
$$

with the same leading coefficient. By uniqueness of the minimal polynomial, all coefficients must be equal, which implies $m h g_{m}=0$, and thus $h=0$. So $Y(s)=0$ and consequently $s \in \mathbb{C}$ unless $Y$ admits a first integral in $\mathbb{K}$.

Part (b) of Proposition 1 is a direct consequence of part (a) and multiplicativity of the field extension degree. 
4.2. Quadratic extensions. In this subsection we consider the smallest possible degrees of $\mathbb{K}[u]$ and $\mathbb{K}[v]$. Thus in view of Proposition 1 we assume relation (5) and furthermore that $u$ and $v$ in are contained in a degree two extension $\mathbb{F}=\mathbb{K}[u]=\mathbb{K}[v]$ of $\mathbb{K}$. With $u$ of norm one, there exists $g \in \mathbb{K}$ such that

$$
u^{2}+2 g \cdot u+1=0
$$

This relation may be rewritten in the form

$$
(u+g)^{2}=g^{2}-1
$$

which in turn implies

$$
u_{x}=-\frac{u}{u+g} g_{x}, \quad u_{y}=-\frac{u}{u+g} g_{y} .
$$

Moreover

$$
v=a+b u, \quad \text { with } a, b \in \mathbb{K} \text { and } b \neq 0
$$

by Proposition 1.

Using (9) and (12) we obtain

$$
\begin{aligned}
v^{2} & =a^{2}+2 a b u+b^{2} u^{2} \\
& =\left(a^{2}-b^{2}\right)+(2 a-2 g b) b u \\
& =\left(a^{2}-b^{2}-2 a(a-g b)\right)+2(a-g b) v
\end{aligned}
$$

and trace zero forces $a=g b$, therefore

$$
v=b(g+u) .
$$

Proof of Theorem 3. The assertions regarding $u$ and $v$ have already been shown. We first determine the Hamiltonian vector field of $H:=b(u+g)+$ $\log u$. With (11) and (10) one finds

$$
\begin{aligned}
H_{x} & =b_{x}(u+g)+b\left(u_{x}+g_{x}\right)+u_{x} / u \\
& =b_{x}(u+g)+b g g_{x}-g_{x} /(u+g) \\
& =(u+g)^{-1} \cdot\left(b_{x}\left(g^{2}-1\right)+g_{x}(b g-1)\right) \\
& =1 / \sqrt{g^{2}-1} \cdot\left(b_{x}\left(g^{2}-1\right)+g_{x}(b g-1)\right)
\end{aligned}
$$

and a similar expression for $H_{y}$. For the rational vector field

$$
\widehat{X}=\left(\begin{array}{c}
-b_{y}\left(g^{2}-1\right)-g_{y}(b g-1) \\
b_{x}\left(g^{2}-1\right)+g_{x}(b g-1)
\end{array}\right)
$$

there obviously exists a unique (up to a scalar factor) rational function $s$ such that $s \cdot \widehat{X}$ is polynomial with relatively prime entries. Thus part (a) holds, and (b) is satisfied by construction.

To prove part (c), assume that the vector field admits a Darboux first integral. Then Corollary 1 yields a contradiction since the integrating factor from part (b) is not rational. 
Theorem 3 describes all polynomial vector fields which admit an exceptional elementary first integral of type (5) with $u$ and $v$ in a quadratic extension of $\mathbb{K}$. One may start with arbitrary rational functions $g$ (such that $u \notin \mathbb{K})$ and $b \neq 0$. But one has to ascertain that the vector field admits no rational first integral. To verify that there actually exist such vector fields, we discuss two examples.

Example 1. This is known from Example 2 of Prelle and Singer [10]: Let $g=x$ and $b=y$. The vector field

$$
\widehat{X}=\left(\begin{array}{c}
1-x^{2} \\
x y-1
\end{array}\right)
$$

admits the integrating factor $1 / \sqrt{1-x^{2}}$ and the elementary first integral

$$
H(x, y)=y \cdot \sqrt{x^{2}-1}+\log \left(-x+\sqrt{x^{2}-1}\right) .
$$

Prelle and Singer [10] showed by differential-algebraic arguments that no rational first integral exists.

Example 2. The polynomial differential system

$$
\begin{aligned}
& \dot{x}=1-x^{2}, \\
& \dot{y}=1-x^{2}-x y,
\end{aligned}
$$

admits the integrating factor $\left(x^{2}-1\right)^{-3 / 2}$ and the elementary first integral

$$
H=\frac{e^{\frac{y}{\sqrt{x^{2}-1}}}}{x+\sqrt{x^{2}-1}}
$$

(or equivalently $\log H=\frac{y}{\sqrt{x^{2}-1}}-\log \left(x+\sqrt{x^{2}-1}\right)$ ). In order to show that this system has no Darboux first integral, it is again sufficient to prove there is no rational first integral. We proceed here using an argument different from Prelle and Singer.

Obviously the only stationary points of the system are $(1,0)$ and $(-1,0)$, and the lines defined by $x \pm 1=0$ are invariant algebraic curves with cofactors $-x \pm 1$, respectively. We show that no other invariant algebraic curves exist. An orbit of the system which contains the point $\left(x_{0}, y_{0}\right)$ with $x_{0}^{2} \neq 1$ is contained in the zero level set of the function

$$
e^{\frac{y}{\sqrt{x^{2}-1}}}-\eta \cdot\left(x+\sqrt{x^{2}-1}\right)
$$

for some constant $\eta \neq 0$. This level set is connected but not an algebraic curve. To see this, note that the level set intersects the line $x=x_{0}$ in infinitely many points $\left(x_{0}, y_{0}+2 \pi i \cdot k \sqrt{x_{0}^{2}-1}\right), k$ any integer. Hence it contains a Zariski-dense subset of the line but not the line itself.

To summarize: The only possible rational first integrals have the form $(x-1)^{m}(x+1)^{n}$, with integers $m$ and $n$. But any integer linear combination $m \cdot(-x+1)+n \cdot(-x-1)$ of the cofactors is nonzero unless $m$ and $n$ are both zero. Therefore, no rational first integral exists. 
4.3. Cubic extensions. In this subsection we discuss exceptional vector fields $X$ which admit a first integral as in (5), with $u$ of norm one and $v$ of trace zero in a cubic extension $\mathbb{F}=\mathbb{K}[u]=\mathbb{K}[v]$ of $\mathbb{K}$. (Recall Proposition 1.) Throughout we assume that the vector field admits no rational first integral. Then the vector field admits a unique (up to a scalar factor) integrating factor $\mu$. We first show Proposition 2, which states that $\mathbb{F}=\mathbb{K}[\mu]$ with $\mu^{3} \in \mathbb{K}$.

Proof of Proposition 2. By Propositions 5 and 6 we have an integrating factor $\mu \in \mathbb{F} \backslash \mathbb{K}$, whence $\mathbb{F}=\mathbb{K}[\mu]$ for degree reasons. The minimal polynomial of $\mu$ thus has degree three, and the splitting field of this minimal polynomial has degree three or six over $\mathbb{K}$. For degree three then we obtain the assertion from Proposition 6. There remains to exclude the possibility of degree six. The minimal polynomial implies a relation

$$
\mu^{3}=r+s \cdot \mu+t \cdot \mu^{2}
$$

with $r, s, t \in \mathbb{K}$. We will lead the assumption that $s \neq 0$ or $t \neq 0$ to a contradiction. First we obtain

$$
\begin{aligned}
\mu^{4} & =r \cdot \mu+s \cdot \mu^{2}+t\left(r+s \cdot \mu+t \cdot \mu^{2}\right) \\
& =r t+(r+t s) \cdot \mu+\left(s+t^{2}\right) \cdot \mu^{2}
\end{aligned}
$$

and from this, with some routine computations,

$$
\begin{aligned}
\mu^{6}= & \left(r+s \cdot \mu+t \cdot \mu^{2}\right)^{2} \\
= & r^{2}+r t^{3}+2 r s t+\left(t^{2}(r+t s)+2 r s+2 s^{2} t\right) \cdot \mu \\
& +\left(s^{2}+3 t^{2} s+t^{4}+2 r t\right) \cdot \mu^{2}
\end{aligned}
$$

Since $\mu^{6} \in \mathbb{K}$ by Proposition 6 , the coefficients of $\mu$ and of $\mu^{2}$ must vanish in this relation. After some rearrangement we get

$$
\begin{array}{ll}
\left(t^{2}+2 s\right)(r+t s) & =0 ; \\
2 t(r+t s)+s^{2}+s t^{2}+t^{4} & =0 .
\end{array}
$$

In case $r+t s=0$ we have $s^{2}+s t^{2}+t^{4}=0$ which implies

$$
s=\beta t^{2}, \text { with } \beta^{2}+\beta+1=0,
$$

and furthermore $r=-\beta t^{3}$. From the minimal polynomial we now obtain

$$
(\mu / t)^{3}=(\mu / t)^{2}+\beta(\mu / t)-\beta,
$$

whence $\mu / t \in \mathbb{C}$ and $\mu \in \mathbb{K} ;$ a contradiction.

In case $s=-t^{2} / 2$ the coefficient for $\mu^{2}$ yields $r=t^{3} / 8$ and

$$
(\mu / t)^{3}=(\mu / t)^{2}-\frac{1}{2}(\mu / t)+\frac{1}{8}
$$

again we obtain the contradiction $\mu \in \mathbb{K}$.

For the further discussion of the degree three case it seems convenient to start with the integrating factor $\mu$ (satisfying a relation (6)) and look for 
elements of norm one (resp. trace zero) in $\mathbb{K}[\mu]$. As in (7) consider a general element

$$
w:=a+b \cdot \mu+c \cdot \mu^{2} \in \mathbb{F} ; \quad a, b, c \in \mathbb{K}
$$

With a primitive third root $\zeta$ of unity, the conjugates of $w$ are

$$
\begin{aligned}
\sigma(w) & =a+\zeta b \cdot \mu+\zeta^{2} c \cdot \mu^{2}, \\
\sigma^{2}(w) & =a+\zeta^{2} b \cdot \mu+\zeta c \cdot \mu^{2} .
\end{aligned}
$$

Lemma 4. (a) The element $w$ given by (7) has norm equal to

$$
N(w)=c^{3} \cdot g^{2}-3 a b c \cdot g+b^{3} \cdot g+a^{3}
$$

and trace equal to $3 a$.

(b) Every element $u \in \mathbb{F}$ of norm one has a representation

$$
u=\sigma(w) / w
$$

with some nonzero $w$.

Proof. Part (a) follows via a straightforward computation from the relation

$$
N(w)=w \cdot \sigma(w) \cdot \sigma^{2}(w)
$$

while part (b) is a direct consequence of Hilbert's Theorem 90 (see Lang [8]).

Lemma 5. Let $w$ be a nonzero element of $\mathbb{F}$, and $u=\sigma(w) / w$. Then

$$
\frac{u_{x}}{\mu \cdot u}=\frac{1}{N(w)} R_{11}+\frac{\mu}{N(w)} \cdot R_{12}
$$

with rational functions $R_{11}$ and $R_{12}$. The explicit form of $R_{12}$ is

$$
\begin{aligned}
R_{12} & \left.=b c^{2} g \cdot\left(c_{x} / c-b_{x} / b+\frac{1}{3} g_{x} / g\right)\right) \\
& +a b^{2} \cdot\left(b_{x} / b-a_{x} / a+\frac{1}{3} g_{x} / g\right) \\
& +a^{2} c \cdot\left(a_{x} / a-c_{x} / c-\frac{2}{3} g_{x} / g\right)
\end{aligned}
$$

Likewise,

$$
\frac{u_{y}}{\mu \cdot u}=\frac{1}{N(w)} R_{21}+\frac{\mu}{N(w)} \cdot R_{22}
$$

with rational functions $R_{21}$ and $R_{22}$. The explicit form of $R_{22}$ is obtained from the one for $R_{12}$ by replacing all partial derivatives with respect to $x$ by the corresponding partial derivatives with respect to $y$.

Proof. We first note that $\mu^{3}=g$ implies

$$
\mu_{x}=\frac{1}{3} \mu g^{-1} g_{x}
$$

and similarly for $\mu_{y}$.

Starting from $u=\sigma(w) / w$ one finds

$$
u_{x}=\frac{(\sigma(w))_{x} \cdot w-w_{x} \cdot \sigma(w)}{w^{2}}
$$


and furthermore

$$
\begin{aligned}
u_{x} / u & =\left((\sigma(w))_{x} \cdot w-w_{x} \cdot \sigma(w)\right) /(w \cdot \sigma(w)) \\
& =\sigma^{2}(w) \cdot\left((\sigma(w))_{x} \cdot w-w_{x} \cdot \sigma(w)\right) / N(w) .
\end{aligned}
$$

From here on the assertion follows by straightforward computation.

Lemma 6. Let $w \in \mathbb{F}^{*}$ be as in (7), and $u=\sigma(w) / w$. Moreover let $r, s \in \mathbb{K}$ and

$$
v:=r \cdot \mu+s \cdot \mu^{2} \in \mathbb{F}
$$

an element of trace zero. The elementary function

$$
H:=v+\log u
$$

is a first integral of a nonzero rational vector field with integrating factor $\mu$ if and only if the identities

$$
\begin{aligned}
& s_{x}+\frac{2}{3} s g^{-1} g_{x}+R_{12} / N(w)=0 \\
& s_{y}+\frac{2}{3} s g^{-1} g_{y}+R_{22} / N(w)=0
\end{aligned}
$$

are satisfied.

Proof. One has

$$
\begin{aligned}
v_{x} & =r_{x} \mu+s_{x} \mu^{2}+(r+2 s \mu) \mu_{x} \\
& =\left(r_{x}+\frac{1}{3} r g^{-1} g_{x}\right) \cdot \mu+\left(s_{x}+\frac{2}{3} s g^{-1} g_{x}\right) \cdot \mu^{2}
\end{aligned}
$$

from (13). The remaining assertions are then clear from the previous computations.

Proof of Proposition 3. Parts (a) and (b) follow directly from the two Lemmas above, since every derivation of $\mathbb{K}$ is a $\mathbb{K}$-linear combination of $\partial / \partial x$ and $\partial / \partial y$. As for part (c), assume that $s_{x} \neq 0$ without loss of generality, and let $y_{0} \neq 0$ such that $x \mapsto s\left(x, y_{0}\right)$ is not constant. Abbreviating $\widehat{h}(x):=h\left(x, y_{0}\right)$ for any function of two variables, Lemma 6 shows that

$$
\widehat{s}^{\prime}+\frac{2}{3} \widehat{s} \cdot \widehat{g}^{\prime} / \widehat{g}+\widehat{R}_{12} / \widehat{N(w)}=0 .
$$

Given $w$ and $g$, this is a linear differential equation for $\widehat{s}$, with general solution

$$
S=\widehat{g}^{-2 / 3} \cdot Q, \quad Q^{\prime}=\widehat{g}^{2 / 3} \cdot \widehat{R}_{12} \widehat{\widehat{N(w)}},
$$

obtained by variation of constants. The difference of two particular solutions is a scalar multiple of $\widehat{g}^{-2 / 3}$, hence not rational. Therefore, if there exists a rational solution $\widehat{s}$ then it is unique.

We refrain from an in-depth discussion of possible rational solutions for the system of partial differential equations given in Lemma 6. But we show by way of an example that generally no rational solutions exist. 
Example 3. We keep notation and terminology from above. Let $g$ be arbitrary with $g_{x} \neq 0, a \in \mathbb{C}^{*}$ and set

$$
w:=a+\mu, \quad N(w)=g+a^{3}, \quad R_{12}=\frac{a}{3} \cdot \frac{g_{x}}{g} .
$$

For this choice of $w$ there exists no rational function $s$ such that the first condition in Lemma 6 can be satisfied.

To verify this, choose $y_{0}$ suitably. We show there exists no rational function $\widehat{s}$ such that

$$
\widehat{s}^{\prime}+\frac{2}{3} \widehat{s} \cdot \frac{\widehat{g}^{\prime}}{\widehat{g}}+\frac{a}{3} \cdot \frac{\widehat{g}^{\prime}}{\widehat{g} \cdot\left(\widehat{g}+a^{3}\right)}=0 .
$$

The general solution of this differential equation is given by

$$
S=\widehat{g}^{-2 / 3} \cdot q, \quad q^{\prime}=-\frac{a}{3} \cdot \frac{\widehat{g}^{2 / 3} \cdot \widehat{g}^{\prime}}{\widehat{g} \cdot\left(\widehat{g}+a^{3}\right)}=-\frac{a}{3} \cdot \frac{\widehat{g}^{\prime}}{\widehat{g}^{1 / 3} \cdot\left(\widehat{g}+a^{3}\right)} .
$$

A substitution leads to the indefinite integration problem

$$
-\frac{a}{3} \cdot \int \frac{d t}{t^{1 / 3}\left(t+a^{3}\right)}=-a \cdot \int \frac{z}{z^{3}+a^{3}} d z
$$

with one more substitution $z=t^{1 / 3}, t=z^{3}$ and $d t=3 z^{2} d z$. With $\zeta$ a primitive third root of unity, one has

$$
-a \cdot \frac{z}{z^{3}+a^{3}}=\frac{1}{3}\left(\frac{1}{z+a}+\frac{\zeta^{2}}{z+\zeta a}+\frac{\zeta}{1+\zeta^{2} a}\right),
$$

and therefore (upon back-substitution) one has a local representation

$$
q=\frac{1}{3} \cdot\left(\log \left(\widehat{g}^{1 / 3}+a\right)+\zeta^{2} \log \left(\widehat{g}^{1 / 3}+\zeta a\right)+\zeta \log \left(\widehat{g}^{1 / 3}+\zeta^{2} a\right)\right)+\text { const. }
$$

Since $q$ is a transcendental function for any value of the constant, we are done.

The question whether nontrivial positive examples exist must be left unresolved here. We were not able to construct an example of a polynomial vector field which admits an exceptional first integral with $u$ and $v$ in some degree three extension of $\mathbb{K}$ but not a rational first integral.

\section{ApPENDiX}

In this section we recall some familiar notions, terminology and facts.

Definition 2. Let $U$ be a nonempty open subset of $\mathbb{C}^{2}$.

We say that a nonconstant (possibly multivalued) function $H: U \longrightarrow \mathbb{C}$ is a first integral of the vector field $X$ on $U$ if and only if the relation $X(H)=0$ holds on $U$.

Given some nonconstant analytic function $g$ on $U$, the analytic vector field

$$
X_{g}:=-\frac{\partial g}{\partial y} \frac{\partial}{\partial x}+\frac{\partial g}{\partial x} \frac{\partial}{\partial y}
$$


is called the Hamiltonian vector field of $g$. By construction it admits the first integral $g$.

We say that a nonconstant (multivalued) function $\mu: U \longrightarrow \mathbb{C}$ is an integrating factor of the vector field $X$ on $U$ if and only if $X(\mu)=-\left(P_{x}+\right.$ $\left.Q_{y}\right) \mu$ on $U$; in other words $\operatorname{div}(\mu \cdot X)=0$.

If $H$ is a first integral of the vector field $X$, then there is a unique integrating factor $\mu$ satisfying

$$
\mu P=\frac{\partial H}{\partial y} \quad \text { and } \quad \mu Q=-\frac{\partial H}{\partial x} .
$$

Next we recall some properties of invariant algebraic curves.

Definition 3. Let $f \in \mathbb{C}[x, y]$ be an irreducible polynomial. The algebraic curve defined by $f(x, y)=0$ is an invariant algebraic curve of the polynomial vector field (1) if there exists a polynomial $K \in \mathbb{C}[x, y]$ (called the cofactor of $f$ ) such that

$$
X f=K f .
$$

Such a condition is necessary and sufficient for the curve to be an invariant set, thus a union of trajectories of the vector field (1).

If the vector field (1) admits an integrating factor (3) then the algebraic curves defined by $f_{i}=0$ are invariant. Moreover, if $K_{i}$ is the cofactor of $f_{i}$, respectively, then

$$
\sum d_{i} K_{i}+\operatorname{div} X=0
$$

We are furthermore concerned with elementary first integrals of (1). Thus consider the differential field $\mathbb{K}=\mathbb{C}(x, y)$ with derivations $\partial / \partial x$ and $\partial / \partial y$. Every derivation of $\mathbb{K}$ has the form $Y=R \frac{\partial}{\partial x}+S \frac{\partial}{\partial y}$ with rational $R$ and $S$.

Definition 4. (a) An extension field $\mathbb{L}$ of $\mathbb{K}$ is called elementary if there is a finite tower of extension fields $\mathbb{K}=\mathbb{L}_{0} \subset \mathbb{L}_{1} \subset \cdots \subset \mathbb{L}_{n}=\mathbb{L}$ such that $\mathbb{L}_{i+1}$ is obtained from $\mathbb{L}_{i}$ by adjoining an algebraic element, an exponential (i.e. an element $w$ such that $Y(w) / w \in \mathbb{L}_{i}$ for some derivation $Y$ ) or a logarithm (i.e. an element $w$ such that $Z(w)=$ $Z(a) / a$ for some $a \in \mathbb{L}_{i}$ and some derivation $Z$ ).

(b) An extension field $\mathbb{L}$ is called Liouvillian over $\mathbb{K}$ if there is a finite tower of extension fields $\mathbb{K}=\mathbb{L}_{0} \subset \mathbb{L}_{1} \subset \cdots \subset \mathbb{L}_{n}=\mathbb{L}$ such that $\mathbb{L}_{i+1}$ is obtained from $\mathbb{L}_{i}$ by adjoining an algebraic element, an integral element (i.e. an element $w$ such that $Z(w)=$ a for some $a \in \mathbb{L}_{i}$ ), or an exponential. Every element of $\mathbb{L}$ is called a Liouvillian function of two variables.

One may regard such field extensions as abstract objects, but in the present work we realize them (locally, on suitable open subsets of $\mathbb{C}^{2}$ ) as analytic functions of two variables. The class of Liouvillian functions obviously contains the class of elementary functions, and the inclusion is proper. 


\section{ACKNOWLEDGEMEnTS}

The first author is partially supported by an AGAUR grant number 2014SGR-568, the grants FP7-PEOPLE-2012-IRSES 318999 and 316338, and the MINECO/FEDER grant UNAB13-4E-1604.

The first two authors are also supported by MINECO grant MTM201340998-P. The second author is partially supported by an AGAUR grant 2014SGR-504, and a MINECO grant MTM2012-31714. The third author acknowledges the hospitality of the Mathematics Department at UAB when this manuscript was prepared.

\section{REFERENCES}

[1] A. Bostan, G. Chéze, T. Cluzeau, J.-A. Weil: Efficient algorithms for computing rational first integrals and Darboux polynomials of planar polynomial vector fields. Math. Comp. 85 (2016), 1393-1425.

[2] J. Chavarriga, H. Giacomini, J. Giné, J. Llibre: Darboux integrability and the inverse integrating factor. J. Differential Eqs. 194 (2003), 116-139.

[3] C. Christopher: Liouvillian first integrals of second order polynomial differential equations. Electronic J. Differential Eqs. 1999, no. 49 (1999), 1-7.

[4] C. Christopher, J. Llibre, C. Pantazi, S. Walcher: Inverse problems for multiple invariant curves. Proc. Roy. Soc. Edinburgh 137A (2007), 1197-1226.

[5] C. Christopher, J. Llibre, C. Pantazi, S. Walcher: Darboux integrating factors: Inverse problems. J. Differential Eqs. 250 (2011), 1-25.

[6] C. Christopher, J. Llibre, C. Pantazi, S. Walcher: Inverse problems in Darboux theory of integrability. Acta Appl. Math. 120 (2012), 101-126.

[7] J. Giné, J. Llibre: A note on the Liouvillian integrability. J. Math. Anal. and Appl. 387 (2012), 1044-1049.

[8] S. Lang: Algebra, Springer, New York 2002.

[9] J. Llibre and C. Pantazi, Polynomial differential systems having a given Darbouxian first integrals, Bull. Sci. Math. 128 (2004), 775-788.

[10] M. J. Prelle and M. F. Singer: Elementary first integrals of differential equations. Trans. Amer. Math. Soc. 279 (1983), 613-636.

[11] M. Rosenlicht: Liouville's theorem on functions with elementary integrals. Pacific J. Math. 24 (1968), 153-161.

[12] M. Rosenlicht: On Liouville's theory of elementary functions. Pacific J. Math. 65 (1976), 485-492.

[13] M.F. Singer: Liouvillian first integrals of differential equations. Trans. Amer. Math. Soc. 333 (1992), 673-688.

${ }^{1}$ Departament de Matemàtiques, Universitat Autònoma de Barcelona, Edifici C, 08193 Bellaterra, Barcelona, Catalonia, Spain

E-mail address: jllibre@mat.uab.cat

3 Departament de Matemàtica Aplicada I, Universitat Politècnica de Catalunya, (EPSEB), Av. Doctor Marañón, 44-50, 08028 Barcelona, Catalonia, SPAIN

E-mail address: chara.pantazi@upc.edu

4 Lehrstuhl A für Mathematik, RWTh Aachen, 52056 Aachen, Germany

E-mail address: walcher@mathA.rwth.aachen.de 